\title{
Preparing a laboratory for radioanalytical emergency response
}

\author{
By J. Bennett*, C. J. Webb and S. Isch \\ Connecticut Department of Public Health, 10 Clinton Street, Hartford CT, 06106, USA
}

(Received November 25, 2009; accepted in revised form November 12, 2010)

\section{Radioanalytical laboratory design / \\ Radioanalytical emergency response / Emergency preparedness}

\begin{abstract}
Summary. As the state of the nation's ability to respond to a radiological event is examined, it has become apparent that both capacity and capability are lacking. Department of Homeland Security National Planning Scenario \#11 is designed to address the planning activities for the response to an attack using radiological dispersal devices. The scenario details show that the cleanup activity will take several years, and that there will be between 360000 and 1000000 environmental samples in the first year. Based on existing capacity and capabilities it would take four to six years to analyze the samples generated at the lower end of the sample range.

The Environmental Protection Agency (EPA) has been given responsibility for the remediation activities following a radiological event, and has awarded cooperative agreements to several laboratories to start the process of developing capacity and capabilities. The Connecticut Department of Public Health Laboratory (DPHL) was awarded one of the cooperative agreements. The DPHL has started activities to further those goals by investigating and implementing procedures to ensure that samples with activity higher than normal background can be processed safely, as well as implementing more rapid methods for radiochemical analysis. The DPHL already served as the primacy radiochemistry laboratory for several New England states and thus had a solid foundation to build upon. The DPHL has taken a process flow approach in preparing for radiological emergency response and recommends that radioanalytical laboratories that are reviewing their roles in such a response:
\end{abstract}

- Ensure that their Nuclear Regulatory Commission licenses allow for appropriate radioisotope types and activities;

- Develop procedures and processes to ensure that samples with higher activities can be processed safely, with due regard for sample screening and aliquanting samples;

- Provide for enhanced radioanalytical contamination control, with careful consideration of sample flow and breaking the laboratory into zones with controlled access;

- Address personnel safety by enhancing training, adding real time dosimetry to exposure monitoring protocols, and reviewing personal protective equipment and hygiene protocols with staff;

- Develop plans for spills and decontamination, as well as for increased monitoring of laboratory areas;

- Plan for secure sample storage;

- Exercise the plan.

\footnotetext{
*Author for correspondence (E-mail: Jack.Bennett@ct.gov).
}

\section{Introduction}

Radioanalytical response has become an area of increasing awareness and concern in the United States and throughout the world. The purpose of this paper is to give a laboratory perspective on this important subject, both to aid the understanding of emergency response partners and to assist other laboratories in their planning for response to radiological incidents. As the state of readiness in the ability of the nation to respond to a radiological event is examined, it becomes apparent that there are significant gaps that need to be addressed by adding analytical capability and capacity. Several Senate hearings took place in 2007 on the ability of the United States to respond to a radiological event. During the November of 2007 hearing before the Senate Committee on Homeland Security and Governmental Affairs titled "Not a Matter of 'If' but of 'When': the Status of US Response Following an RDD Attack", experts from federal agencies testified about the efforts that their agencies were making to respond. A common theme of the testimony was that they were aware of the problem, but were constrained by lack of resources. Senator Coleman commented during the hearing: "It can happen and we must be prepared to deal with it." [1-3]. Since that time other hearings and reports have continued to focus on radiological response activities including a report by the Commission on the Prevention of Weapons of Mass Destruction Proliferation and Terrorism called World at Risk which concluded that the risks from WMD (including nuclear) were growing faster than our ability to respond [4].

In order to plan and prepare for potential disaster scenarios, the Department of Homeland Security released a series of potential types of events (both naturally occurring and terror related) to guide planning efforts in March of 2006 [5]. There are twelve National Planning Scenarios (NPS) covering possibilities ranging from pandemic flu to hurricanes to cyber attack. NPS Number 11 deals with a radiological dispersal device (RDD). The scenario supposes that there would be three simultaneous explosions in three geographically close major urban areas. As a result of the explosions, there would be between 100000 to 300000 people exposed and 20000 to 60000 of those exposed would have detectable levels of contamination. Additionally, in the first year of the cleanup effort there would be between 360000 and 1000000 environmental samples generated. The food supplies could be impacted as well. At the time of the Senate hearing, it was estimated that that it would take four years to 
complete the analysis of 100000 clinical samples and four to six years to analyze 350000 environmental samples.

It has been over three years since that hearing, and some progress has been made in both field and laboratory preparedness. The ASTM has developed a standard addressing the field component of radiological response [6]. The Food and Drug Administration (FDA) has set up cooperative agreements with four laboratories in its Food Emergency Response Network (FERN) and has a number of noncooperative agreement laboratories as well. The Centers for Disease Control and Prevention (CDC) is developing rapid radioanalytical methods and is seeking funding to set up a radiological component to its Laboratory Response Network (LRN). The Environmental Protection Agency (EPA) has had two rounds of funding, as a result of which it has set up five-year cooperative agreements with four laboratories, three in the first round of funding and a fourth laboratory in the second round of funding. The rest of this paper will describe the experiences of one state, Connecticut, in implementing the EPA Radioanalytical Capacity and Capability Enhancement Cooperative Agreement.

\section{Background}

EPA issued a Request for Proposal (RFP) to fund laboratories under a cooperative agreement in October 2007. The Connecticut Department of Public Health Laboratory (DPHL) applied because it had a mature radioanalytical program, with significant engagement of numerous emergency response partners on the regional, state, and local levels. It serves as the Safe Drinking Water Act Primacy radiochemistry laboratory for Connecticut and Massachusetts, is the ingestion pathway response laboratory in case of an accident at the nuclear power plant complex in Connecticut, performs routine monitoring around the power plants, and is a member of the EPA RADNET network. We proposed to identify and address the bottlenecks that limit the ability to respond rapidly in the case of a radiological incident and to develop procedures and processes to ensure that samples with higher than background activity could be processed safely in the laboratory. There were two major bottlenecks that were identified: time consuming sample preparation and a lack of analytical instrumentation. EPA indicated in the request for applications that they would supply a significant amount of instrumentation, including alpha spectroscopy. EPA would provide two high purity germanium systems, two gas flow proportional systems, one liquid scintillation counter, and one alpha spectroscopy (alpha spec) system with sixteen detectors. This mix of equipment would complement (and nearly double) the amount of instrumentation at the Connecticut laboratory because, with the exception of the alpha spec, the laboratory was already well equipped with radioanalytical instrumentation. To address the capability portion of the cooperative agreement, Connecticut has hired a $\mathrm{PhD}$ level analytical chemist to develop more rapid methods of sample preparation and to serve as technical lead on the project. EPA has also provided on-site training for other staff and laboratory partners to aid in surge capacity.

The time to prepare the laboratory for safely processing samples for radioanalytical response is prior to an event.
Event response requires that a laboratory have a Nuclear Regulatory Commission (NRC) license (which is not a requirement for laboratories performing Safe Drinking Water Act radiochemical analyses), an enhanced radiation safety plan, enhanced radioanalytical contamination control, and enhanced contamination monitoring. These have all been implemented at the DPHL, and will be discussed below.

\section{Design considerations for a new radiological response laboratory}

The initial cooperative agreements were awarded in September 2008 to Connecticut, Texas, and Washington. Even prior to the release of the RFP, Connecticut was already designing a new state-of-the-art Public Health Laboratory, and emergency response (including radiological response) was integral to the design process. After the cooperative agreement was awarded, the instrumentation included in the agreement was incorporated into the design, and the requirements for contamination control and safety were further developed.

As the design process was occurring several key concepts were considered and developed. The first consideration was that emergency response would require that the laboratory accept samples with a higher activity than would normally be submitted to a laboratory dealing with typical environmental samples. Three possible solutions to address this requirement were discussed. They were: to construct two separate facilities, to do everything in one laboratory, or to provide for separate areas in one facility. The DPHL design team selected the last option because it provided a reasonable level of assurance that higher activity samples would not contaminate the low-level laboratory. A layout of the laboratory design is presented as Fig. 1.

The second consideration was to determine what level of sample activity to accept in the laboratory. The answer to that consideration somewhat depends on the decision made above. Unless two separate facilities were to be constructed, contamination control becomes a concern. A balance needs to be struck so that samples of a high enough activity to be useful in providing information to guide the response activities and yet not potentially contaminate the facility are accepted. Connecticut has decided that a $2.5 \mathrm{mRem} / \mathrm{h}$ per sample limit will allow us to provide the data users with results that will allow them to make appropriate response decisions and keep potential contamination under control. This criterion was developed after consultation with the Connecticut Department of Environmental Protection (the radiation control agency in the state), and allows the laboratory to meet the FDA Derived Intervention Levels (DILS) [7] and the EPA Protective Action Guidelines (PAG) [8].

As was mentioned above, the design decision was to construct a separate area for emergency response activities within the footprint of the new CT DPHL laboratory. The implementation of the decision was to design an emergency response suite. The suite would consist of a sample receipt lab located on the outside wall of the building (Fig. 1B), a small sample preparation (prep) lab (Fig. 1 - D), and a small instrument room (Fig. 1-C). For local events, it would be likely that samples would be brought directly to the laboratory by the sampling team. To prevent potential 


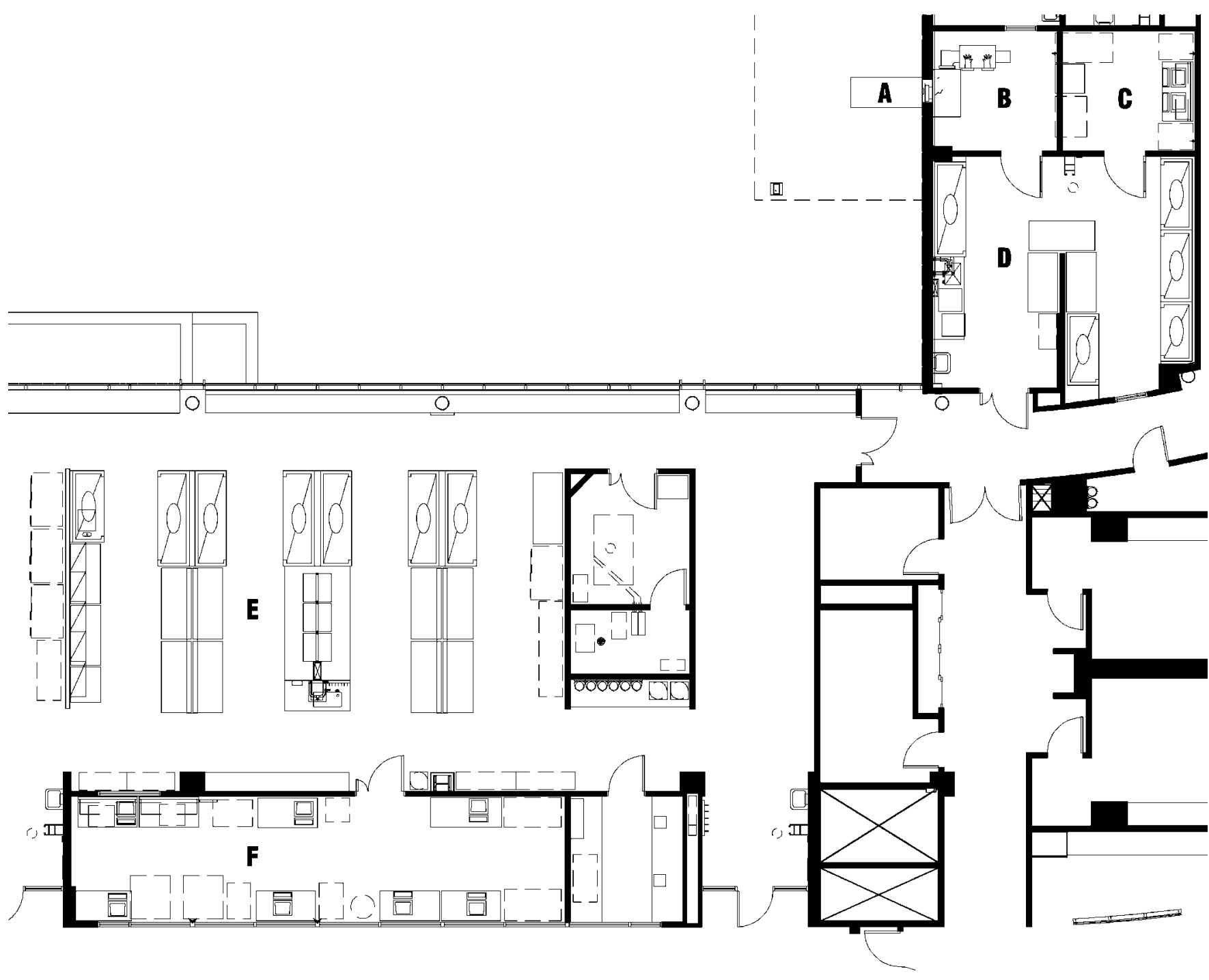

Fig. 1. Connecticut DPHL design schematic. A - Table for DOT type screening; B - sample receipt lab; C - screening counting room; D - sample preparation lab; E - low level sample prep lab; F - counting room.

contamination from being inadvertently brought into the laboratory by the samplers, it was decided to construct an event sample receipt area outside the laboratory. It would be a heated semi-enclosed area, much like a bus shelter. There would be a stainless steel table (Fig. 1 - A) where Department of Transportation (DOT) type screening could be performed. After the preliminary screening, the samples would be transferred to the sample receipt laboratory directly into a hood via a pass-through. If samples were shipped to the laboratory using a common carrier, the samples would still be brought to the same event sample receipt area. It is important to note that coolers or sample bottles should not be opened during this preliminary screening and that this screening is only for external assessment of the containers.

The pass-through into the hood would be large enough to accept a 40 quart cooler, and the hood would have a recess in the floor of the hood to allow the cooler to sit low enough so that it could be opened and inspected while protecting the worker. Since the design of the facility encompasses emergency response, the hood will also be connected to a glove box for screening for chemical hazards. If the samples were to be submitted in small coolers, the recess could be covered with a plate to provide a work surface. The room exhaust is separate from the rest of the facility exhaust, and is passed through HEPA filtration.

After the samples are unpacked (and any container screening performed), the samples will be brought to the preparation lab. The preparation lab is to be equipped with several hoods, including a polypropylene fume hood, a perchloric acid hood, a radioisotope hood and a traditional fume hood. The design also includes a lead-lined cabinet for shortterm storage of samples. The lab will also be equipped with muffle furnaces and equipment for reducing the particle size of solids. Preliminary sample pretreatments to reduce the level of activity in the sample aliquants can be done in this laboratory. In a response situation, having a prep lab separate from the low level lab that is used primarily for water samples is important so that contamination will not be spread to the low level lab. The third room of the suite is a small counting room containing a liquid scintillation counter, a large and a small sodium iodide ( $\mathrm{NaI}[\mathrm{Tl}])$ detector, and a gas proportional counter for large air filters. This instrumentation will be used for preliminary screening of samples to characterize the level of activity. The air pressure in this room is positive compared to the preparation lab to reduce the potential for airborne contamination. Ideally, this room should be larger 
than is currently designed to allow better separation of the gamma instrumentation from the gas proportional counters, but the balancing of laboratory functions and available space did not allow for this to happen.

The new CT DPH laboratory was designed so that there will be a short transit distance from the emergency response suite to the low-level preparation laboratory (Fig. 1 - E) and counting room (Fig. 1-F), and the corridors can be barricaded to prevent non-radiation workers from traversing the area.

At a very early stage in the design process for the new CT DPH laboratory, a decision was made that (as much as possible) the design would be based around the concept of an "open laboratory" (i.e. a minimum number of walled off laboratories) in order to allow flexibility for future operational changes. This presented some challenges in designing the low-level radiochemistry laboratory because of the unique nature of radioanalytical analyses. As will be discussed below, contamination control is of paramount importance in this type of work. Ideally, for emergency response operations, the various functions (e.g. sample prep, sample storage and sample counting) would be performed in segregated, shielded areas with strict access control for each of the areas. However, the ideal situation was not practicable, so the design of the low level laboratory was a compromise that incorporated some engineering controls with administrative provisions (even taking into account that some prealiquanting and/or preparation of samples would take place in the all hazards laboratory). Some of the design elements included:

- The counting room was located at the opposite end of the laboratory from the hood where sample preparation was to occur, and the airflow across the laboratory was designed to flow from the counting room towards the hoods.

- Walls were constructed at each end of the laboratory to provide some level of separation between the radiochemistry prep lab and the rest of the laboratories in the wing of the building (although the doorways between laboratory sections were still open).

- The radiochemistry laboratory was located adjacent to the inorganic chemistry laboratory (which had hoods similar to those in the radiochemistry laboratory) so that potentially higher activity samples could be processed there to avoid potential contamination issues in the lowlevel radiochemistry laboratory.

- Traffic can be made unidirectional by blocking off aisles between the bench islands.

- The count room was designed with the gamma instrumentation as far away from the gas proportional counters and alpha specs as possible.

\section{Contamination control}

The first line of defense for any laboratory considering radioanalytical response activities is to prevent unchecked contamination from entering the laboratory. Sample screening can be an important element of this process. Screening can (and should) be done at more than one stage of the analytical process. The first screening should be done on the packaging before the sample enters the building. If this is not possible, it should be done as early in the receipt process as is practicable. This type of screening is often referred to as Department of Transportation (DOT) screening. It is often done either by using an instrument such as a Geiger-Muller (GM) counter and passing the probe over the surface of the shipping container or swiping the surface of the container (with something like maslin) and checking the swipe with the GM counter. This process will use either radiation dose (millirem/hour $[\mathrm{mrem} / \mathrm{h}]$ ) or gross activity in counts per minute (CPM) as a quick check to verify that samples with high activity either do not enter the facility or are treated with appropriate care, and will only assess gamma or high energy beta particle activity.

After the initial screening, further characterization of the level of activity should be performed. This can be accomplished through the use of gas proportional counting or liquid scintillation counting. These will require opening the sample, and should be done (if possible) in a fume hood. Additionally, an aliquant can also be screened with a $\mathrm{NaI}(\mathrm{Tl})$ detector to guide the further analysis of the samples for gamma photons. If a laboratory does not have a dedicated area where the screening can be performed, it should identify an area in the laboratory where as much of the screening or preparation of the aliquant for screening can be performed. Detailed information about screening considerations can be found in the Radiological Laboratory Sample Screening Analysis Guide for Incidents of National Significance [9].

As was mentioned above, contamination control is an essential component of radiological incident response. Contamination control can be accomplished by breaking the laboratory into zones. At the very least, there should be two zones. One zone should be for people in the laboratory who do not have radiation safety training, and thus samples that could contain radioactivity would not be allowed in that zone. The second zone would be for people who were trained and would have access to rad samples. The second zone could be further subdivided between areas for higher activity samples and lower activity samples. There needs to be some way to control access to each of the zones. The method of control may be as simple as cordoning off one zone from the other or something more involved like key card access.

It is likely that portions of the sample preparation process will have to be done in the low level prep lab, and a good way to minimize the potential of contaminating the low level lab is to use the smallest sample size that will meet the Data Quality Objectives (DQO's). This can be based on the screening information, which will be used to perform aliquanting to the appropriate level of activity. When actually performing the aliquanting, it is important to consider process flow. The work area should be divided into three areas. One area would be for staging the higher activity samples, the second area would be where the work on the sample was actually performed, and the third area would be for the reduced activity aliquanted portion of the samples. The workflow would be from higher levels of activity to lower levels of activity with the higher activity portion never entering the lower activity area and vice-versa. In an instance where it may not be possible to aliquant a sample to a low 
enough activity to process in the low level radiochemistry lab, it may be necessary to do digestion in the emergency response laboratory (or an area of the laboratory separate from the low level radiochemistry laboratory), and a portion of the digestate with the appropriate activity would be transported to the low level lab.

The institution of screening and activity control through the appropriate reduction of sample activity by sample size reduction will not only minimize the physical contamination of the laboratory, it will also protect the detectors used in the sample analysis. Especially in the later stages of an event, it will be extremely important to have low background in the detectors in order to meet DQO's established by Incident Command established for that particular stage of the event. In some instances, it may be a good idea to protect the detector with a removable plastic bag as an additional assurance to protect the detector from contamination.

Another type of contamination to consider is the cross contamination of samples through improperly cleaned glassware. At the very least it would be important to clean glassware used for higher activity samples separately from glassware used for low activity samples, but it would be worthwhile to consider using disposable labware wherever possible. There may be some considerations that have to be addressed prior to disposal, but they should have minimal impact.

It is also imperative to address the personnel aspects of contamination control, because they are even more important than the physical plant considerations. It may be worthwhile to add real time dosimetry to exposure monitoring protocols. Prior to sample receipt, it is important to review personal protective equipment (PPE) and personal hygiene protocols with staff, to encourage staff to change gloves more frequently than usual, to use step-off pads when going from areas where higher activity samples are processed to cleaner areas, and if possible, to use frisking stations as well. The step-off pads should be changed frequently, and the frisking station can be used not only to monitor personnel but also to verify that samples can move to the lower activity area.

No discussion of contamination control would be complete without mentioning that spill response and decontamination should be addressed. The response plan for small spills in a laboratory should not be unduly difficult to implement because the level of activity (although higher than the laboratory typically handles) should not require extraordinary measures. Some typical types of supplies might include: a squirt bottle with a chelating agent, disposable wiping material (like maslin), large baggies or some type of bin and a properly marked waste container. After the spill is cleaned up, the area should be surveyed to verify that the cleanup was adequate. The cleanup process can be made easier if the laboratory has sealed seamless flooring, uses plastic backed absorbent paper on benches and hoods that are used for sample preparation, and uses a strippable coating on the insides of hoods. An effective way of surveying the floor is to use a mop with maslin (or something similar), mopping the entire area and surveying the mophead. If there is no detectable contamination, the entire area can be considered decontaminated. If there is detectable contamination, the large areas should be broken down into grids and each grid is surveyed individually. This procedure can also be used post event prior to returning the laboratory to normal operations.

Additionally, it will be important to increase the level of monitoring in the laboratory itself. The frequency of monitoring will need to increase, but the number of areas to be monitored will need to be increased as well. It would also be desirable to periodically monitor the general access areas to ensure that inadvertent contamination was not introduced there. The action levels for the area monitoring may be different for each stage of an event.

When performing the screening, it is important to be able to distinguish whether or not the instrument reading is different from the background. The Multi-Agency Radiation Survey and Site Investigation Manual (MARSSIM) [10] provides information to help with that determination, using the principles established by Currie [11]. The two parameters that are important are the level (in counts) at which a reading can be considered above background $\left(L_{\mathrm{C}}\right)$ and the level (in counts) that can be seen by a detector with a $95 \%$ confidence of detecting the contamination when it is present (with only a $5 \%$ probability of a false positive) $\left(L_{\mathrm{D}}\right)$. It is important to remember that these are net counts above background. These terms are defined below:

$$
\begin{aligned}
& L_{\mathrm{C}}=2.33 \sqrt{\frac{\text { Backgroundcpm }}{\text { Minutes }}} \\
& L_{D}=3+4.65 \sqrt{\frac{\text { Backgroundcpm }}{\text { Minutes }}}
\end{aligned}
$$

During radiation emergency response activities, contamination is considered to be present if the survey meter reading is two to three times the background reading. The laboratory should consider the implications of using the higher limit if it decides that it is appropriate for their operation. In normal operations, the CTDPHL background ranges between (approximately) 30 to $40 \mathrm{cpm}$ (using a pancake probe), and the quarterly monitoring badges worn by the staff are below the detection limits of the badges.

It will be necessary to plan for sample storage. It is impractical to build sufficient storage space in a laboratory prior to an event, so it is important to have a plan to address the need. One possibility is to procure trailers that can be used for sample storage, as is the plan in Connecticut. The trailers need to be secured to prevent unauthorized people from accessing the trailer, and also may need to be environmentally controlled to ensure proper storage conditions. It is also important to inventory the amount of material stored so that the total activity does not get so high that the dose to people entering the trailer to access the samples is excessive. The trailers should be stored far enough away from the laboratory that they do not impact operations.

\section{Radiological response planning in existing laboratories}

This paper has primarily discussed considerations to be addressed when building a new facility. However, the same concepts can be implemented (in many cases) in existing facilities, as is illustrated by Connecticut's experience. For the 
advance planning highlighted above, it will be necessary to critically examine existing procedures to institute changes that will work in a given context. It may involve cooperation between different areas of the laboratory, but it can be done. In Connecticut, the planning process for the new laboratory design along with its experience with response activities for nuclear power plant accidents provided a framework for making decisions to modify the current facility and institute similar procedures to enhance contamination control.

The Connecticut Public Health Laboratory was fortunate to have an entrance off the parking lot that is not routinely used. The laboratory's policy is that everyone enters the building through the front entrance (for security purposes), so that the unused entrance can be set up as a receiving area for event samples. Tables can be set up across the entrance for samplers to drop off samples and to perform DOT screening on the samples. Samplers will not enter the laboratory, thus preventing inadvertent contamination from occurring.

After preliminary triage, the cooler can be carried up to the second floor. During an event, the door on the first floor would be cordoned off, and the stairwell above the second floor would be cordoned off. The stairwell exits onto the second floor adjacent to the west door of a laboratory that would be used for initial unpacking of the cooler (in a hood) and preliminary screening and sample preparation. After any initial screening, aliquanting and/or preparation, the sample would exit through the east door of the laboratory and be transported across the hall to the main radiochemistry preparation lab. After any further preparation, the sample would be transported to one of two counting rooms. The counting room at the west end of the radiochemistry laboratory would (in general) be used for higher activity samples and the counting room at the east end of the radiochemistry laboratory would be used for lower activity samples.

The advance planning required of laboratories to implement a radiological response plan necessitates consideration of contamination control, personnel safety and monitoring, sample storage, and decontamination. In addition other operational changes are likely to be necessary. The testing that is performed in the laboratory where the initial screening would take place would have to be discontinued, and there would be interruption of testing that is performed in adjacent laboratories because corridors would need to be blocked off. However, in an existing building there may be some less than obvious items to address. For example, many existing laboratories have floor drains and tile floors. It may be necessary to cover the floor of the laboratory with plastic sheeting to aid cleanup activities that would result from an accidental spill of a contaminated sample. It is important to exercise the plan to uncover these unforeseen contingencies.

Finally, the advance planning (for both new and existing facilities) must include ways to process a large number of samples quickly and provide data with well-defined uncertainties. Guidance on providing data with well-defined uncertainties cam be found in the Multi-Agency Radiological Laboratory Analytical Protocols Manual [12]. EPA has developed and published several guidance documents to help the laboratory with the proper approach to prioritization, screening and analysis [13-15] because the traditional methods of sample analysis will not provide the throughput necessary to effectively determine the extent of contamination or effectiveness of remediation. Rapid methods need to be developed and validated prior to being used. EPA is also in the process of developing guidance documents to help plan for operations and contamination control during an incident $[16,17]$.

As laboratories continue to develop plans to accept samples from an emergency response perspective, it is important to realize that an event requiring a radioanalytical response will cause unique challenges that must be addressed before samples arrive at the laboratory. Even laboratories that currently perform testing on low activity samples may not be ready to appropriately handle higher activity samples because the handling practices for higher activity samples are not practiced on a routine basis. The topics addressed in this paper can serve as a starting point for developing and refining policies and procedures to protect both the health of the laboratory personnel as well as the laboratory infrastructure.

Acknowledgment. Portions of this work were supported through a cooperative agreement with the US Environmental Protection Agency (XA-83405901).

\section{References}

1. Government Accountability Office (GAO). Combating Nuclear Terrorism: Preliminary Observations on Preparedness to Recover from Possible Attacks Using Radiological or Nuclear Materials. GAO Report 09-996T (14 September 2009).

2. Staff Report of the Subcommittee on Investigations and Oversight of the House Science and Technology Committee. Radiological Response: Assessing Environmental and Clinical Laboratory Capabilities. Report release date: 25 October 2007. Downloaded from http://democrats.science.house.gov/Media/File/Commdocs/ hearings/2007/oversight/25oct/Rad\%20Labs\%20FINAL\%20STA FF\%20REPORT.pdf on 5 November 2009.

3. Hearing Before the Subcommittee on Investigations and Oversight of the House Science and Technology Committee. Radiological Response: Assessing Environmental and Clinical Laboratory Capabilities. Serial Number 110-67. Hearing date: 25 October 2007. Downloaded from http://frwebgate.access.gpo.gov/cgi-bin/g etdoc.cgi?dbname $=110 \_$house_hearings\&docid=f:38340.wais.pdf on 5 November 2009.

4. Graham, B., Talent, J.: World at Risk: The Report of the Commission on the Prevention of Weapons of Mass Destruction Proliferation and Terrorism. Vintage Books, New York (December 2008), downloaded from http://documents.scribd.com/docs/15bq1nr19ae rfu0yu9qd.pdf on 5 November 2009.

5. Department of Homeland Security: Planning Scenarios Created for Use in National, Federal, State and Local Preparedness Activities (Final Version 21.3), March (2006), available at LLIS.gov.

6. ASTM International: Standard Practice for Radiological Emergency Response (E2601-08), downloaded from ASTM.org on 9 June 2009.

7. Food and Drug Administration: Accidental Radioactive Contamination of Human Food and Animal Feeds, Recommendations for State and Local Agencies, August 1998, accessed at http://www.fda.gov/downloads/MedicalDevices/DeviceRegulation andGuidance/GuidanceDocuments/UCM094513.pdf on 20 October 2010.

8. EPA: Manual of Protective Action Guides and Protective Actions for Nuclear Incidents, 1992, accessed from http://www.epa.gov/ rpdweb00/rert/pags.html on 20 October 2010.

9. United States Environmental Protection Agency (USEPA): Radiological Laboratory Sample Screening Analysis Guide for Incidents of National Significance. EPA 402-R-09-008 (June 2009).

10. EPA: Multi-Agency Radiation Survey and Site Assessment Manual, June 2001, accessed from http://www.epa.gov/rpdweb00/ marssim/obtain.html on 20 October 2010. 
11. Lloyd, A. C.: Limits for qualitative detection and quantitative determination: application to radiochemistry. Anal. Chem. 40, 586593 (1968).

12. EPA: Multi-Agency Radiological Laboratory Analytical Protocols Manual, accessed from http://www.epa.gov/rpdweb00/marlap/ manual.html on 20 October 2010.

13. United States Environmental Protection Agency (USEPA): Radiological Sample Analysis Guide for Incidents of National Significance - Radionuclides in Air. EPA 402 R-09-007 (June 2009).

14. United States Environmental Protection Agency (USEPA): Method Validation Guide for Qualifying Methods Used by Radio- logical Laboratories Participating in Incident Response Activities. EPA 402-R-09-006 (June 2009).

15. United States Environmental Protection Agency (USEPA): Radiological Sample Analysis Guide for Incidents of National Significance - Radionuclides in Water. EPA 402-R-07-007 (Jan. 2008).

16. United States Environmental Protection Agency: Guide for Radiological Laboratories for the Control of Radiological Contamination and Radiation (Draft - September 2009).

17. United States Environmental Protection Agency: Guide for Radiological Laboratories for the Identification, Preparation and Implementation of Core Operations for Radiological Incident Response (Draft - August 2009). 